\title{
Inhomogeneous Vasodilatory Responses of Rat Tail Arteries to Heat Stress: Evaluation by Synchrotron Radiation Microangiography
}

\author{
Eriko KuWABARA, Fujiya FuruYAmA*, Kunihisa ITO, Etsuro TANAKA, Naoichiro HatTAN, \\ Hisanori FUJIKURA, Koji KIMURA, Takako GOTO, Takashi HAYASHI, Hiroyuki TAIRA, \\ Yoshiro SHINOZAKI, Keiji UMETANI ${ }^{\dagger}$, Kazuyuki HYODO ${ }^{\ddagger}$, Kenkichi TANIOKA ${ }^{\S}$, Ryo MOCHIZUKI", \\ Toshiaki KAWAI", Shirosaku KOIDE, and Hidezo MORI ${ }^{\#}$ \\ Departments of Surgery, Physiology, and Cardiology, and Center for Regenerative Medicine, \\ Tokai University School of Medicine, Isehara, 259-1193 Japan; \\ * Department of Neurophysiology and Brain Sciences, Nagoya City University Graduate School \\ of Medical Sciences, Nagoya, 467-8601 Japan; \\ † Japan Synchrotron Radiation Research Institute, Sayo-gun, Hyogo, 679-5198 Japan; \\ ¥ High Energy Accelerator Research Organization, Tsukuba, 305-0801 Japan; \\ $\S$ NHK Science and Technical Research Laboratories, Tokyo, 157-8510 Japan; \\ "NHK Engineering Services Inc., Tokyo, 150-0042 Japan; \\ " Hamamatsu Photonics K.K., Hamamatsu, 430-8587 Japan; \\ and Department of Cardiac Physiology, National Cardiovascular \\ \# Center Research Institute, Suita, 565-8565 Japan
}

\begin{abstract}
Tail blood flow is crucial for dissipating body heat in rats. Angiographies are convenient tools to evaluate tail circulation. However, conventional angiographies do not have sufficient sensitivity or spatial resolution for small vessels. Recently, we developed a novel microangiographic system using monochromatic synchrotron radiation and a high-definition video camera system. Here, we report an evaluation of rat tail circulation under heat stress using the synchrotron radiation microangiographic system. We performed an experiment using the microangiography of the caudal artery before and after heating up WKAH/HkmSlc rats to rectal temperature of $39^{\circ} \mathrm{C}$. The images were digitized and temporal subtraction was performed, and the diameters of caudal arteries were evaluated. After
\end{abstract} heating, the medial caudal artery was markedly dilated $(320 \pm 53$ to $853 \pm 243 \mu \mathrm{m}$ in diameter, $p<0.001$ ), while no significant change was observed in the lateral caudal arteries $(139 \pm 42$ to $167 \pm 73 \mu \mathrm{m})$ and segmental anastomosing vessels. The heat stress allowed for visualization of the superficial caudal arteries with a diameter of approximately $60 \mu \mathrm{m}$, not visible prior to heating. Thus, synchrotron radiation microangiography demonstrated that the rat tail possessed dual sets of arteries; one set was highly sensitive to heat-induced vasodilation (medial caudal artery and superficial caudal arteries) and the other set was less sensitive (lateral caudal arteries and segmental anastomosing vessels). [Japanese Journal of Physiology, 52, 403-408, 2002]

Key words: angiography, heat stress, peripheral circulation, vascular control, vasodilation.

$\mathrm{V}$ (i.e., skin) plays an important role in dissipating body heat in humans [1], as vasodilation in the tail does in rats $[2,3]$. The rat tail has one large artery, the medial caudal artery, and many small arteries. The small arteries are distributed both in the superficial and deep regions in the tail.

In vivo analyses of regional blood flow and/or va-

Received on June 11, 2002; accepted on August 15, 2002

Correspondence should be addressed to: Hidezo Mori, Department of Cardiac Physiology, National Cardiovascular Center Research Institute, Suita, 565-8565 Japan. Tel: +81-6-6833-5012 ext 2530, Fax: +81-6-6835-5416, E-mail: hidemori@ri.ncvc.go.jp 
soreactivity in the rat tail have been done using various techniques (e.g., plethysmography [4-6], thermomonitoring [2], microsphere [7], laser Doppler [7], and X-ray angiography [8, 9]). Thermo-monitoring and laser Doppler methods reflect the blood flow only in superficial regions, and the microsphere method often fails to evaluate arteriovenous anastomoses. Angiography is able to evaluate even deep vessels consecutively. However, conventional X-ray angiography has neither the sensitivity needed to detect small amounts of iodine contrast material nor the spatial resolution to quantitate the diameters of the small vessels. For example, quantification of the diameters is impossible in vessels with a diameter of less than $300 \mu \mathrm{m}$ [10]. Recently, we developed a novel microangiographic system with high contrast resolution and high spatial resolution using monochromatic synchrotron radiation as an X-ray source and a high-definition video camera system as a detector [11]. This system allowed depiction of small vessels in the leg (collateral arteries) [12], intestinal organs (vasa recta and their submucosal communications) [13], brain (small branches arising from the canine circle of Willis) [10], heart (penetrating transmural artery) [14], and cancer tissue (angiogenic vessels) [15], and depiction of small branches (down to the fifth order) of the pancreatic duct [13]. This system using analog images also demonstrated that the heat stress caused vasodilation in the medial caudal artery but not in the abdominal cutaneous artery in rats [9].

The purpose of this study was to evaluate vasodilatory responses to heat stress of rat tail arteries distinguishing between superficial and deep regions using an animated synchrotron radiation microangiographic system and digital image processing with higher time-, spatial-, and contrast-resolutions.

\section{MATERIALS AND METHODS}

Animals. Fourteen WKAH/HkmSlc rats (Japan SLC Inc., Hamamatsu, Japan) weighing 350 to $450 \mathrm{~g}$ were used. The animals were anesthetized with intravenous infusion of ketamine hydrochloride $(1.0 \mathrm{mg} /$ $\mathrm{kg} / \mathrm{min}$ ) via a catheter placed in the femoral vein after intraperitoneal injection of $125 \mathrm{mg} / \mathrm{kg}$ of the same drug. All procedures were conducted in accordance with the Guiding Principles for the Care and Use of Animals approved by the Council of the Physiological Society of Japan.

Angiography. The fundamental design of our synchrotron radiation angiographic system has already been reported (Fig. 1) [9-16]. Briefly, monochromatic synchrotron radiation with an energy level of $33.3 \mathrm{keV}$ was obtained with a silicon crystal from beamlines NE5 and BL-14 in the High Energy Accelerator Research Organization, Tsukuba, Japan. The contrast images were formed on a fluorescent screen in which a $20 \times 20 \mathrm{~mm}$ area was scanned at 15 or 30 frames/s by a high-definition video camera with 1125 TV lines [17, 18], and then stored as a digital image in a memory system $(20 \times 20 \mathrm{~mm}$ area, $1,024 \times$ 1,024 pixels, 12-bit/pixel). Time-, spatial-, and contrast-resolutions of the present system have been improved in comparison with those of former resolutions [9]. The present system had a spatial resolution of $25 \mu \mathrm{m}$ (20 line pairs/mm studied with a MTF chart) and a time resolution of 33 or $67 \mathrm{~ms}$. To improve contrast resolution, a temporal subtracted image was created on a computer from the digital images obtained immediately before and during contrast material injection. As for contrast resolution using a vascular phantom (Type 76-700, Nuclear Associates, New York, USA), the minimum size $(0.5 \mathrm{~mm}$ of diameter $)$ vascular phantom with a minimum concentration (2.5 $\mathrm{mg} / \mathrm{ml}$ of iodine) was visualized through an acrylic block with a $75 \mathrm{~mm}$ thickness. Vessel diameter was

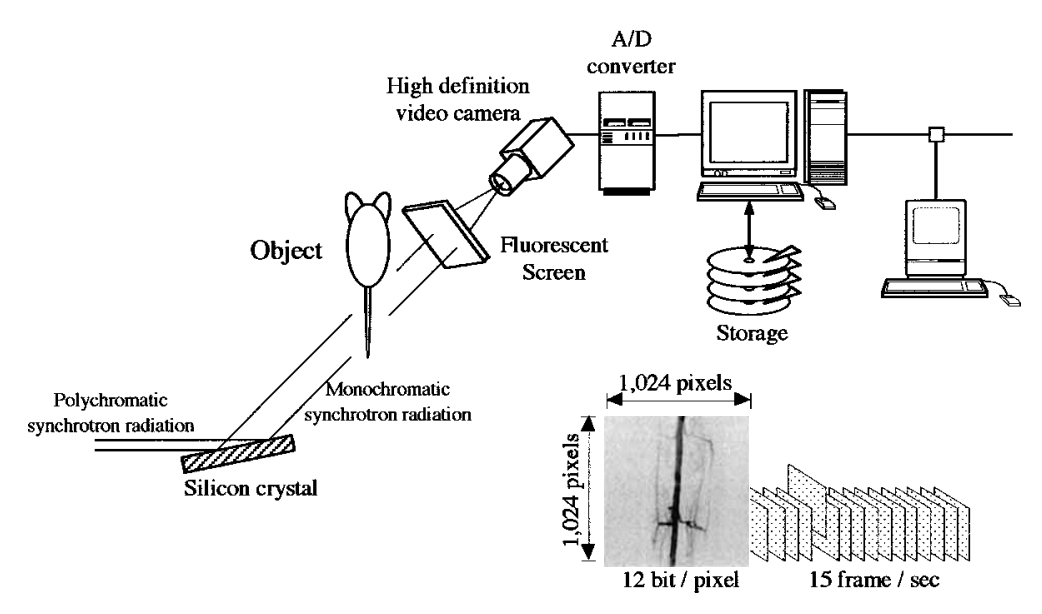

Fig. 1. Schematic presentation of the experimental setup. The contrast-enhanced images were formed on the fluorescent screen scanned by a high-definition video camera. Digital image processing was done on a computer. 
measured on a computer $[10,16]$.

Experimental protocol. After anesthesia, the catheter for injection of the contrast material was placed just above the bifurcation of the common iliac artery from the abdominal aorta via the right femoral artery. The rat was fixed on the acrylic board $(5.0 \mathrm{~mm}$ thick) in standing position for angiography. Arterial pressure was monitored continuously by the catheter placed at the femoral artery, except during the period of the contrast material injection. The arterial systolic pressure ranged from 120 to $140 \mathrm{mmHg}$ throughout the experimental period.

Sequential images of caudal arteries in the proximal portion of the tail were obtained by the injection of non-ionic contrast material $(3 \mathrm{ml} / \mathrm{s}$ for $1 \mathrm{~s}$, Iopamidol, Nihon Schering, Tokyo, Japan) via the arterial catheter. The contrast materials were infused twice, before and after the body temperature was increased from 37 to $39^{\circ} \mathrm{C}$, by heating the body for $15 \mathrm{~min}$ using a hair-dryer. Body temperature was monitored using a thermometer $(52 \mathrm{~K} / \mathrm{J}$ Thermometer, Fluke, USA) inserted $6 \mathrm{~cm}$ into the rectum. The second angiography was performed $20 \mathrm{~min}$ after the first angiography. After completion of the angiographies, histological analysis (hematoxylin and eosin staining) of the tail was performed. The results of the second angiography were independent of the first. This was confirmed by preliminary experiments which indicated that the diameter change of the medial caudal artery was negligible 15 min after the contrast material injection (from $317 \pm 64$ to $331 \pm 70 \mu \mathrm{m}$ at $37^{\circ} \mathrm{C}, n=5, p=0.739$; from $956 \pm 203$ to $899 \pm 124 \mu \mathrm{m}$ at $39^{\circ} \mathrm{C}, n=5, p=0.553$ ).

All data obtained in the present study are expressed as means $\pm \mathrm{SD}$. We applied a paired $t$-test for comparison of the mean values. Differences were considered significant at $p<0.05$.

\section{RESULTS}

The representative synchrotron radiation microangiographic images before and after the increase in body temperature are shown in Fig. 2. The large dumbbelllike structures in the center represent caudal verte-

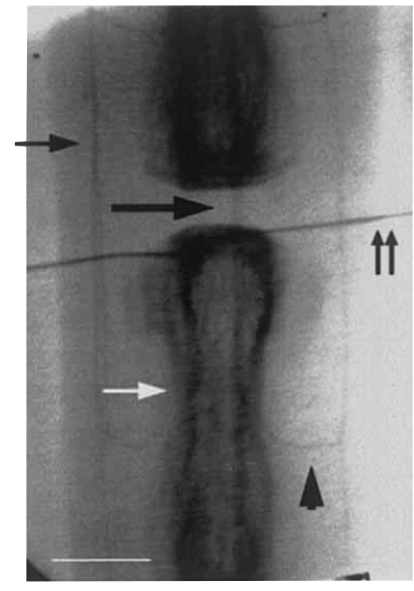

Before heating

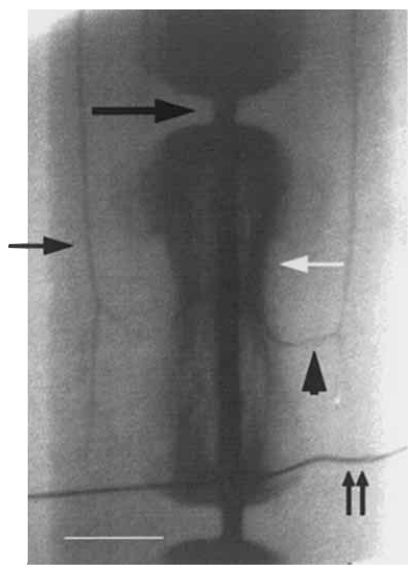

After heating
Fig. 2. Synchrotron radiation angiograms of rat tail before (left) and after heating (right). Large arrows, the medial caudal artery; small arrows, the lateral caudal arteries; arrowheads, segmental anastomosing vessels; white arrow, caudal vertebra; double arrows, reference copper wires with a diameter of $130 \mu \mathrm{m}$. Bar $=3 \mathrm{~mm}$.

brae. The medial caudal artery (large arrows), the lateral caudal arteries (small arrows), and segmental anastomosing vessels (arrowheads) were visualized before heating. After heating, the medial caudal artery was markedly dilated ( $320 \pm 53$ to $853 \pm 243 \mu \mathrm{m}$ in diameter, $p<0.001$ ), while no significant change was observed in the lateral caudal arteries $(139 \pm 42$ to $167 \pm 73 \mu \mathrm{m}, p=0.19$, Table 1$)$. The smallest visible artery was approximately $50 \mu \mathrm{m}$ in diameter as judged from a reference copper wire with a diameter of $130 \mu \mathrm{m}$ (double arrows).

The digital subtraction image processing allowed us to visualize the medial caudal artery, the lateral caudal arteries, and segmental anastomosing vessels more clearly even before heating (Fig. 3A). After heating, small string-like arteries with a diameter of approximately $60 \mu \mathrm{m}$ were visualized beside the lateral caudal arteries and segmental anastomosing vessels (Fig. 3B-H, Table 1). These small arteries are considered to be the superficial caudal arteries based on the anatomical arrangement of rat tail arteries shown in a cross-

Table 1. Vasodilation to heat stress in rat tail arteries.

\begin{tabular}{lcccc}
\hline & $\begin{array}{c}\text { Rectal } \\
\text { temperature }\left({ }^{\circ} \mathrm{C}\right) \\
(n=14)\end{array}$ & $\begin{array}{c}\text { Diameter of the medial } \\
\text { caudal artery }(\mu \mathrm{m}) \\
(n=14)\end{array}$ & $\begin{array}{c}\text { Diameter of the lateral } \\
\text { caudal artery }(\mu \mathrm{m}) \\
(n=14)\end{array}$ & $\begin{array}{c}\text { Number of the superficial } \\
\text { caudal arteries }^{\dagger} \\
(n=5)\end{array}$ \\
\hline Before heating & $37.0 \pm 1.0$ & $320 \pm 53$ & $139 \pm 42$ & $0 \pm 0$ \\
After heating & $38.8 \pm 0.5$ & $853 \pm 243^{*}$ & $167 \pm 73$ & $7.4 \pm 2.4^{*}$ \\
\hline
\end{tabular}

${ }^{\dagger}$ Number of the superficial caudal arteries in one segment was counted. ${ }^{*} p<0.001 \mathrm{vs}$. before heating (paired $t$-test). 


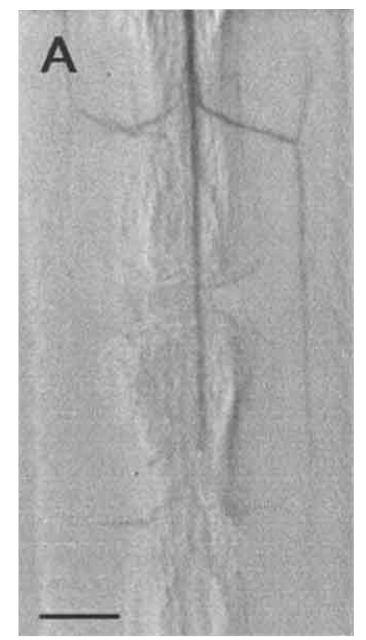

Before heating

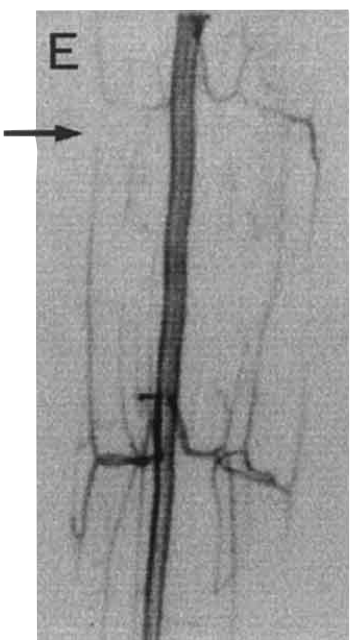

$0.200 \mathrm{sec}$

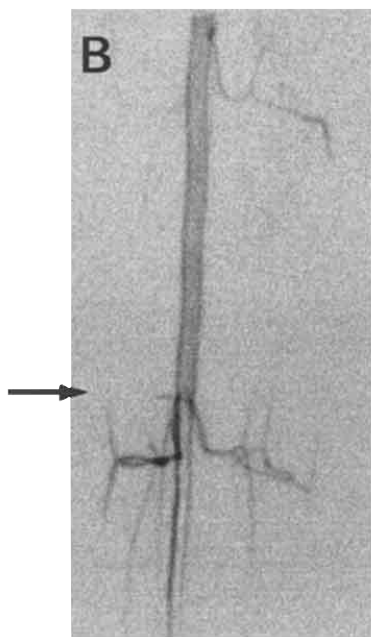

After heating: $0.000 \mathrm{sec}$

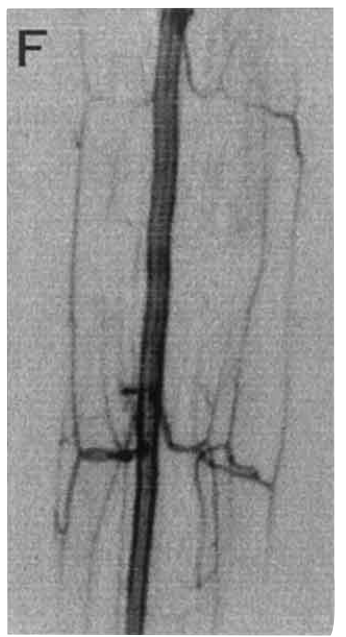

$0.333 \mathrm{sec}$

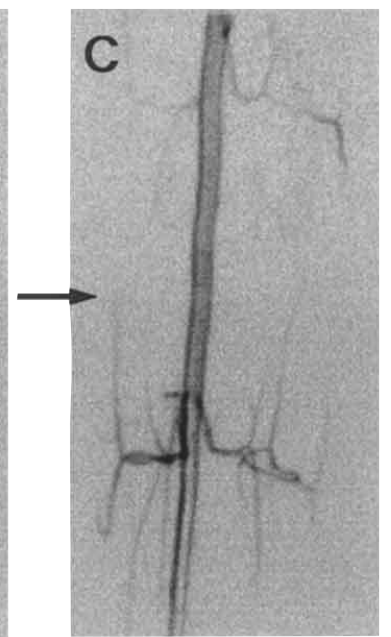

$0.067 \mathrm{sec}$

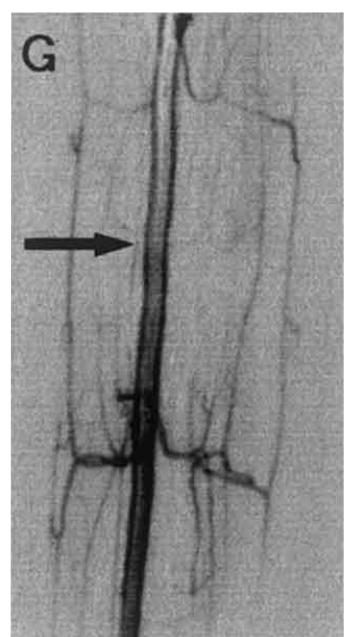

$0.467 \mathrm{sec}$

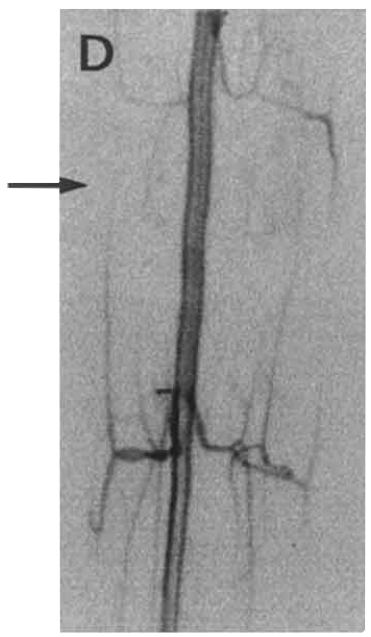

$0.133 \mathrm{sec}$

Fig. 3. Digitally subtracted images of synchrotron radiation angiography of rat tail after heating. A: Before heating. B$\mathbf{H}$ : After heating. Elapsed time from panel B is shown in each panel. Small arrows, the top of the contrast material in the lateral caudal artery; large arrows, the top of the blood flow (the end of the contrast material) in the medial caudal artery. Bar $=3 \mathrm{~mm}$.

section of the tail (arrowheads in Fig. 4). Note that these arteries were not visualized before heating (Fig. $3 \mathrm{~A})$.

Sequential images after heating shown in Fig. $3 \mathrm{~B}-\mathrm{H}$ demonstrate that the direction of the blood flow in the medial caudal artery was from the base to the tip of the tail after heating (large arrows in Fig. 3). It was consistent with the direction of flow before heating (data not shown). On the other hand, in the lateral caudal artery, which was supplied by segmental anastomosing vessels arising from the medial caudal artery, the direction of the flow was not always from the base to the tip (small arrows in Fig. 3). Similar results were observed in the superficial caudal arteries, in which the flow was bidirectional (Fig. 3B-H). Arte- riovenous anastomose-like stainings were not seen in these frames because venous images appear after the arterial images have faded completely (data not shown).

\section{DISCUSSION}

Since monochromatic synchrotron radiation enhances sensitivity for the iodine-contrast material and the high-definition video camera has high sensitivity and high resolution $[10,11]$, we were able to use these systems to perform microangiographies and visualize arteries as small as $50 \mu \mathrm{m}$ in diameter in the rat tail, which has not been demonstrated in vivo.

Synchrotron radiation microangiography demon- 


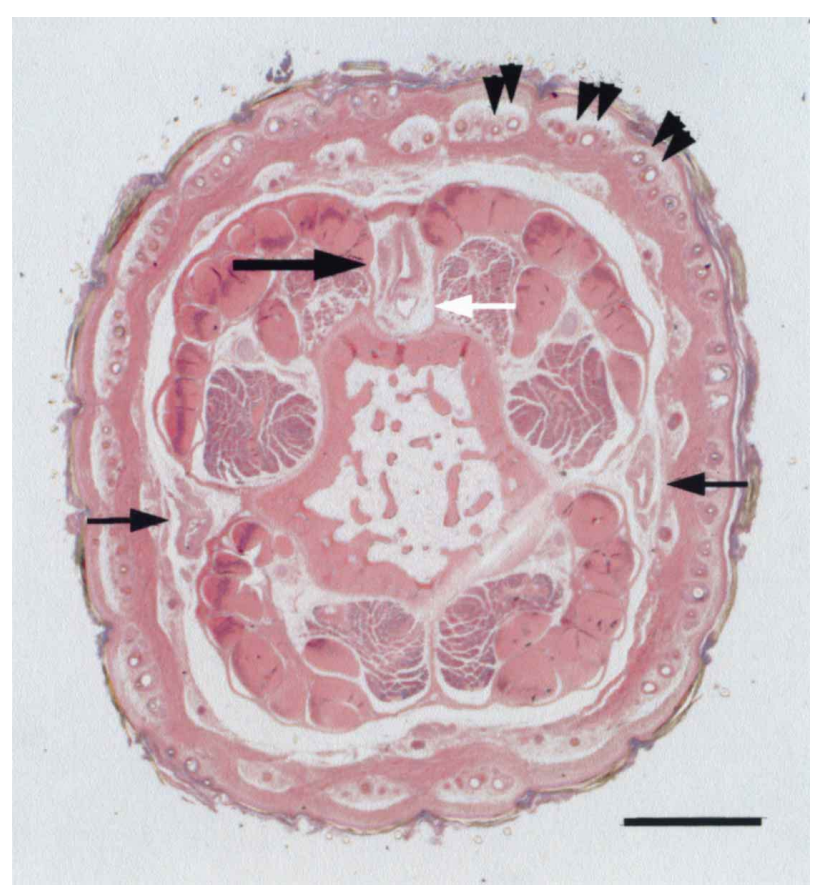

Fig. 4. Cross-section of the joint region of rat tail. The medial caudal artery (large arrow) and the lateral caudal arteries (small arrows) were present in the deep region of the tail. The superficial caudal arteries (arrowheads) were present in the superficial region. White arrow indicates the medial caudal vein. Hematoxylin and eosin stain. Original magnification $\times 1.25$; $B a r=2 \mathrm{~mm}$.

strated that the rat tail possessed a dual set of arteries; It was shown that the medial caudal artery had high vasodilatory responsiveness to heat, but the vasodilatory response of the lateral caudal arteries and segmental anastomosing vessels was small. Furthermore, the superficial caudal arteries, which may remain closed or were very small before heating, showed marked vasodilatation after heating.

It is no surprise that arteries in the superficial region of the tail, such as the superficial caudal arteries, showed high sensitivity to the heat since their function is to perform heat-loss through the tail. The lateral caudal arteries did not respond to the heat, and therefore, their function presumably is not one of heat-loss but of feeding nutrients to the peripheral tissue. As such, they do not give significant flow to the superficial caudal arteries. Inhomogeneous dilation of tail vessels has been reported in rats in response to neuropeptide $\mathrm{Y}$ [7]. The administration of neuropeptide $\mathrm{Y}$ increased total tail blood flow but decreased superficial cutaneous flow in the tail. We previously reported that heat stress did not cause vasodilation in the skin (i.e., superficial inferior epigastric artery) in a rat evaluated by synchrotron radiation angiography [9]. Presumably, the abdominal subcutaneous vessel under the fur does not play an important role in thermoregulation in the rat. Similarly, the medial caudal artery is located in a relatively deep region in the tail and should not play a direct role in heat loss. The underlying mechanism for its marked dilation is a secondary effect of an increased flow to feed dilated superficial caudal arteries since the superficial caudal arteries are primarily fed by the medial caudal artery. Whether or not the medial caudal artery independently responds to heat is unclear.

Arteriovenous anastomoses are abundant in rat tail skin and play an important role in thermoregulation during heat stress [19]. Their diameters were shown to be relatively large since a large fraction of microspheres with a diameter of $15 \mu \mathrm{m}$ injected to the medial caudal artery passed arteriovenous anastomoses [7]. However, we assume that the small arteries in Fig. 3 were not arteriovenous anastomoses but rather were superficial caudal arteries because all arteries were parallel and veins were stained at later stages. Unfortunately, the recording period of the present digital system was too short to demonstrate the venous phase.

Sequential images are advantageous for evaluating the direction of blood flow as shown in Fig. 3, and we were able to demonstrate bidirectional flow in the lateral caudal arteries and superficial caudal arteries.

The authors wish to thank Drs. Yoshiko Shinozaki, Takayuki Hasegawa, and Jobu Ito for their technical work. This work was supported by Grants-in-Aid for Scientific Research (12670064, 13470154, 13470381, 13877114, 14657460, 14657461) from the Ministry of Education, Culture, Sports, Science and Technology; The Science Frontier Program of MECSST; New Energy and Industrial Technology Development Organization; The Research Grants for Cardiovascular Disease (H13C-1) from the Ministry of Health, Labour and Welfare; and the Promotion Fundamental Studies in Health Science of the Organization for Pharmaceutical Safety and Research of Japan. This project was approved as a joint research program of the High Energy Accelerator Research Organization (98G181, 98G194, 98G195, 99G135, 01G182, and 02G160) and of Japan Synchrotron Radiation Research Institute.

\section{REFERENCES}

1. Armstrong CG and Kenney WL: Effects of age and acclimation on responses to passive heat exposure. J Appl Physiol 75: 2162-2167, 1993

2. Shido $O$ and Nagasaka T: Heat loss responses in rats acclimated to heat loaded intermittently. J Appl Physiol 68: 66-70, 1990

3. Sakurada S, Shido O, Fujikake K, and Nagasaka T: Relationship between body core and peripheral temperatures at the onset of thermoregulatory responses in rats. Jpn J Physiol 43: 659-667, 1993 


\section{E. KUWABARA et al.}

4. Rand RP, Burton AC, and Ing T: The tail of the rat, in temperature regulation and acclimatization. Can $J$ Physiol Pharmacol 43: 257-267, 1965

5. Raman ER, Roberts MF, and Vanhuyse VJ: Body temperature control of rat tail blood flow. Am J Physiol 245: R426-R432, 1983

6. O'Leary DS and Johnson JM: Baroreflex control of the rat tail circulation in normothermia and hyperthermia. $J$ Appl Physiol 66: 1234-1241, 1989

7. Heath ME: Neuropeptide $Y$ and $Y 1$-receptor agonists increase blood flow through arteriovenous anastomoses in rat tail. J Appl Physiol 85: 301-309, 1998

8. Young $A A$ and Dawson $\mathrm{NJ}$ : Evidence for on-off control of heat dissipation from the tail of the rat. Can J Physiol Pharmacol 60: 392-398, 1981

9. Ito K, Tanaka E, Mori H, Nakazawa $H$, and Tanino R: A microangiographic technique using synchrotron radiation to visualize dermal circulation in vivo. Plast Reconstr Surg 102: 1128-1133, 1998

10. Tanaka E, Tanaka A, Sekka T, Shinozaki Y, Hyodo K, Umetani K, and Mori H: Digitized cerebral synchrotron radiation angiography: quantitative evaluation of the canine circle of Willis and its large and small branches. Am J Neuroradiol 20: 801-806, 1999

11. Mori H, Hyodo K, Tanaka E, Uddin MM, Yamakawa A, Shinozaki Y, Nakazawa H, Tanaka Y, Sekka T, Iwata Y, Handa S, Umetani K, Ueki H, Yokoyama T, Tanioka K, Kubota M, Hosaka H, Ishikawa N, and Ando M: Smallvessel radiography in situ with monochromatic synchrotron radiation. Radiology 201: 173-177, 1996

12. Takeshita S, Isshiki $T$, Mori $H$, Tanaka E, Eto K, Miyazawa Y, Tanaka A, Shinozaki Y, Hyodo K, Ando M, Kubota M, Tanioka K, Umetani K, Ochiai M, Sato T, and Miyashita $\mathrm{H}$ : Use of synchrotron radiation microangiography to assess development of small collateral arteries in a rat model of hindlimb ischemia. Circulation 95: 805-808, 1997

13. Tanaka $Y$, Mori $H$, Tanaka E, Abe S, Makuuchi $H$,
Nakazawa H, Handa S, Tanioka K, Kubota M, Kumaoka S, Hyodo K, and Ando M: Synchrotron radiation microangiography using an avalanche-type high-definition video camera. In: Medical Applications of Synchrotron Radiation, ed. Ando M and Uyama C, Springer-Verlag, Tokyo, pp 42-53, 1998

14. Mori H, Tanaka E, Hyodo K, Uddin Mohammed M, Sekka T, Ito K, Shinozaki Y, Tanaka A, Nakazawa H, Abe S, Handa S, Kubota M, Tanioka K, Umetani K, and Ando M: Synchrotron microangiography reveals configurational changes and to-and-fro flow in intramyocardial vessels. Am J Physiol 276: H429-H437, 1999

15. Sekka T, Volchikhina SA, Tanaka A, Hasegawa M, Tanaka Y, Ohtani Y, Tajima T, Makuuchi H, Tanaka E, Iwata Y, Sato S, Hyodo K, Ando M, Umetani K, Kubota $\mathrm{M}$, Tanioka $\mathrm{K}$, and Mori $\mathrm{H}$ : Visualization, quantification, and therapeutic evaluation of angiogenic vessels in cancer by synchrotron microangiography. J Synchrotron Rad 7: 361-367, 2000

16. Tanaka A, Mori H, Tanaka E, Mohammed MU, Tanaka Y, Sekka T, Ito K, Shinozaki Y, Hyodo K, Ando M, Umetani K, Tanioka K, Kubota M, Abe S, Handa S, and Nakazawa $\mathrm{H}$ : Branching patterns of intramural coronary vessels determined by microangiography using synchrotron radiation. Am J Physiol 276: H2262-H2267, 1999

17. Tanioka K, Yamazaki J, Shidara K, Taketoshi K, Kawamura T, Hirai T, and Takasaki Y: Avalanche-mode amorphous selenium photoconductive target for camera tube. Adv Electronics Electron Phys 74: 379-387, 1988

18. Umetani K, Ueki H, Takeda T, Itai Y, Mori H, Tanaka E, Mohammed MU, Shinozaki Y, Akisada M, and Sasaki Y: High-spatial-resolution and real-time medical imaging using a high-sensitivity HARPICON camera. J Synchrotron Rad 5: 1130-1132, 1998

19. Gemmell RT and Hales JR: Cutaneous arteriovenous anastomoses present in the tail but absent from the ear of the rat. J Anat 124: 355-358, 1977 